\title{
Hydrogen Bonding as a Construction Element for Bidentate Donor Ligands in Homogenous Catalysis: Regioselective Hydroformylation of Terminal Alkenes
}

Bernhard Breit*, Wolfgang Seiche

Institut für Organische Chemie und Biochemie, Albert-Ludwigs-Universität Freiburg, Germany

\section{Supplementary Material}

\author{
(7 pages)
}

\section{General}

Acetic acid hex-5-enyl ester ${ }^{[1]}$ and undec-10-enoic ${ }^{[2]}$ acid methyl ester were prepared according to literature reported procedures. All other reagents were obtained commercially unless otherwise noted. Reactions were performed using oven-dried glassware under an atmosphere of argon. Airand moisture sensitive liquids and solutions were transferred via syringe. Organic solutions were concentrated under reduced pressure (ca. 20 mbar ) by rotary evaporation. Toluene was distilled from sodium and stored under an atmosphere of argon. Chromatographic purification of products was accomplished using flash chromatography ${ }^{[3]}$ on Macherey-Nagel silica gel 60 (230-400 mesh).

Nuclear magnetic resonance spectra were acquired on a Varian Mercury spectrometer (300 MHz, 121.5 MHz and 75.5 MHz for ${ }^{1} \mathrm{H},{ }^{31} \mathrm{P}$ and ${ }^{13} \mathrm{C}$ respectively) and on a Bruker AMX $400(400 \mathrm{MHz}$ and $100.6 \mathrm{MHz}$ for ${ }^{1} \mathrm{H}$ and ${ }^{13} \mathrm{C}$ respectively) and are referenced internally according to residual protio solvent signals. Data for ${ }^{1} \mathrm{H}$ NMR are recorded as follows: chemical shift $(\delta$, ppm), multiplicity (s, singlet; br s, broad singlet; d, doublet; t, triplet; q, quartet; quint, quintet; m, multiplet), coupling constant (Hz), integration. Data for ${ }^{13} \mathrm{C}$ NMR are reported in terms of chemical shift $(\delta, \mathrm{ppm})$. GC-MS analysis was done on a Varian GC/MS Workstation CP3800/Saturn 2000 on a CP-SIL 5 CB low bleed column. High-resolution mass spectra were obtained on a Finnigan MAT 8200 instrument. Hydroformylation experiments were performed either with an Argonaut Endeavour ${ }^{\circledR}$ Catalyst Screening System or in stainless steel tube autoclaves. Synthesis gas (CO 3.7, $\mathrm{H}_{2}$ 4.3, 1:1) was obtained from Messer.

Caution: All operations involving carbon monoxide must be carried out in a well-ventilated fume hood. Use of a gas-leak detector for carbon monoxide is highly recommended. 


\section{Experimental procedures and characterizations}

\section{2-tert-Butoxy-6-chloro-pyridine (1)}<smiles>CC(C)(C)Oc1cccc(Cl)n1</smiles>

$\mathrm{C}_{9} \mathrm{H}_{12} \mathrm{CINO}$

Mol. Wt.: 185.65

To a solution of $10.00 \mathrm{~g}$ 2,6-dichloro-pyridine (67.6 mmol, $1.0 \mathrm{eq})$ in $150 \mathrm{ml}$ toluene was added $9.10 \mathrm{~g}$ potassium-tert-butylate $(81.1 \mathrm{mmol}, 1.2 \mathrm{eq})$. After heating $6 \mathrm{~h}$ to $80^{\circ} \mathrm{C}$ the suspension was filtered through a plug of silica to remove solid byproducts. The filtrate was concentrated in vacuo and the residual yellow oil was purified via bulb to bulb destillation $\left(200{ }^{\circ} \mathrm{C}, 10^{-1} \mathrm{mbar}\right)$. Compound 1 was obtained as a colorless liquid (11.98 g, 95.5\%).

${ }^{1} \mathrm{H}$ NMR (400 MHz, $\left.\mathrm{CDCl}_{3}, \mathrm{ppm}\right) 7.43(\mathrm{t}, J=7.7 \mathrm{~Hz}, 1 \mathrm{H}), 6.81(\mathrm{~d}, J=7.3 \mathrm{~Hz}, 1 \mathrm{H}), 6.53$ (d, $J$ $=8.2 \mathrm{~Hz}, 1 \mathrm{H}), 1.58(\mathrm{~s}, 9 \mathrm{H})$

${ }^{13} \mathrm{C}\left(100.6 \mathrm{MHz}, \mathrm{CDCl}_{3}, \mathrm{ppm}\right) \quad 163.3,147.7,140.2,115.7,111.3,80.9,28.6$

CHN calcd C: $58.23, \mathrm{H}: 6.52, \mathrm{~N}: 7.54$ found C: $58.27, \mathrm{H}: 6.65, \mathrm{~N}: 7.48$

\section{2-tert-Butoxy-6-diphenylphosphanyl-pyridine (2)}<smiles>CC(C)(C)Oc1cccc(P(c2ccccc2)c2ccccc2)n1</smiles>

$\mathrm{C}_{21} \mathrm{H}_{22} \mathrm{NOP}$

Mol. Wt.: 335.38

To liquid ammonia (ca. $500 \mathrm{ml}$ ) at $-78{ }^{\circ} \mathrm{C}$ was added $5.40 \mathrm{~g}$ sodium $(235.0 \mathrm{mmol}, 2.03 \mathrm{eq})$ during $10 \mathrm{~min}$. The dark blue solution was treated portionwise first with $30.40 \mathrm{~g}$ triphenylphosphin $(115.9 \mathrm{mmol}, 1 \mathrm{eq})$ and then, after stirring for $2 \mathrm{~h}$ at $-78{ }^{\circ} \mathrm{C}$, with $21.50 \mathrm{~g}$ (115.8 mmol, 1 eq) 2-tert-butoxy-6-chloro-pyridine (1). After the addition of $175 \mathrm{ml}$ of tetrahydrofuran the ammonia was allowed to evaporate over night. The residue was quenched with $200 \mathrm{ml}$ of water (dest.), extracted three times with $150 \mathrm{ml}$ diethylether each and dried over $\mathrm{Na}_{2} \mathrm{SO}_{4}$. Evaporation of the solvent in vacuo and recrystallisation of the residual oil from methanol yielded $31.0 \mathrm{~g}(79.8 \%)$ 2-tert-butoxy-6-diphenylphosphanyl-pyridine (2) as a white solid. 
$\mathrm{mp}=77^{\circ} \mathrm{C}$

${ }^{1} \mathrm{H}\left(400 \mathrm{MHz}, \mathrm{C}_{6} \mathrm{D}_{6}, \mathrm{ppm}\right)$ 7.51-7.44 (m, 4H), 7.11-7.04 (m, 6H), $6.91(\mathrm{ddd}, J=8.3,7.3,2.8$

$\mathrm{Hz}, 1 \mathrm{H}), 6.75(\mathrm{dd}, J=7.3,2.8 \mathrm{~Hz}, 1 \mathrm{H}), 6.45(\mathrm{~d}, J=8.3 \mathrm{~Hz}, 1 \mathrm{H})$

${ }^{13} \mathrm{C}\left(100.6 \mathrm{MHz}, \mathrm{C}_{6} \mathrm{D}_{6}, \mathrm{ppm}\right) \quad 164.1\left(\mathrm{~d}, J_{\mathrm{C}, \mathrm{P}}=9.7 \mathrm{~Hz}\right), 160.5,138.0\left(\mathrm{~d}, J_{\mathrm{C}, \mathrm{P}}=5.8 \mathrm{~Hz}\right), 137.6(\mathrm{~d}$, $\left.J_{\mathrm{C}, \mathrm{P}}=11.7 \mathrm{~Hz}\right), 134.7\left(\mathrm{~d}, J_{\mathrm{C}, \mathrm{P}}=18.9 \mathrm{~Hz}\right), 128.9,128.7\left(\mathrm{~d}, J_{\mathrm{C}, \mathrm{P}}=7.2 \mathrm{~Hz}\right), 121.4\left(\mathrm{~d}, J_{\mathrm{C}, \mathrm{P}}=24.7\right)$, $112.2,79.6,28.5$

${ }^{31} \mathrm{P}\left(121.5 \mathrm{MHz}, \mathrm{C}_{6} \mathrm{D}_{6}, \mathrm{ppm}\right) \quad-3.1$

CHN calcd C: 75.21, H: 6.61, N: 4.18 found C: 74.98, H: 6.57, N: 4.09

\section{6-Diphenylphosphanyl-1H-pyridin-2-one (6-DPPon) (3)}<smiles>O=c1cccc(P(c2ccccc2)c2ccccc2)[nH]1</smiles>

$\mathrm{C}_{17} \mathrm{H}_{14} \mathrm{NOP}$

Mol. Wt.: 279.27

$9.70 \mathrm{~g}$ (28.92 mmol) 2-tert-butoxy-6-diphenylphosphanyl-pyridine (2) was dissolved in $100 \mathrm{ml}$ of conc. formic acid which has been saturated with argon. After stirring for 30 min the solution was diluted with $120 \mathrm{ml}$ water (dest.). The precipitate was collected by filtration, washed with $30 \mathrm{ml}$ aqueous formic acid (2:1, v/v) and dried. 6-Diphenylphosphanyl-1H-pyridin-2-on (3) was obtained as a white solid (5.6 g, 69.3\%). The combined aqueous formic acid solutions were concentrated in vacuo and the residue was recrystallized from aceton. This yielded another $1.8 \mathrm{~g}$ (22.2\%) of 6-diphenylphosphanyl-1H-pyridin-2-on (3).

$\operatorname{mp} 187^{\circ} \mathrm{C}$

${ }^{1} \mathrm{H}\left(400 \mathrm{MHz}, \mathrm{CDCl}_{3}, \mathrm{ppm}\right) \quad 9.29$ (br s, $\left.1 \mathrm{H}\right), 7.45-7.25(\mathrm{~m}, 11 \mathrm{H}), 6.51(\mathrm{~d}, J=9.0 \mathrm{~Hz}, 1 \mathrm{H}), 6.20$ $(\mathrm{t}, J=6.3 \mathrm{~Hz}, 1 \mathrm{H})$

${ }^{13} \mathrm{C}\left(100.6 \mathrm{MHz}, \mathrm{CDCl}_{3}, \mathrm{ppm}\right) \quad 163.8,146.6\left(\mathrm{~d}, J_{\mathrm{C}, \mathrm{P}}=26.2 \mathrm{~Hz}\right), 140.5\left(\mathrm{~d}, J_{\mathrm{C}, \mathrm{P}}=7.3 \mathrm{~Hz}\right), 133.9$ $\left(\mathrm{d}, J_{\mathrm{C}, \mathrm{P}}=20.4 \mathrm{~Hz}\right), 132.6\left(\mathrm{~d}, J_{\mathrm{C}, \mathrm{P}}=10.2 \mathrm{~Hz}\right), 130.2,129.3\left(\mathrm{~d}, J_{\mathrm{C}, \mathrm{P}}=7.3 \mathrm{~Hz}\right), 120.7,114.0\left(\mathrm{~d}, J_{\mathrm{C}, \mathrm{P}}\right.$ $=21.8)$

${ }^{31} \mathrm{P}\left(121.5 \mathrm{MHz}, \mathrm{CDCl}_{3}, \mathrm{ppm}\right) \quad-9.8$

CHN calcd C: 73.11, H: 5.05, N: 5.02 found C: 72.97, H: 5.18, N: 4.73 


\section{Phenyl-carbamic acid hex-5-enyl ester (4)}

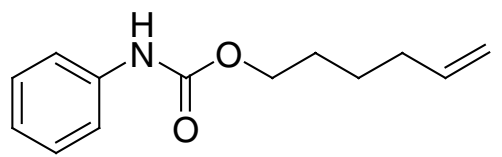

$\mathrm{C}_{13} \mathrm{H}_{17} \mathrm{NO}_{2}$

Mol. Wt.: 219.28

To a solution of $2.54 \mathrm{~g}$ hex-5-en-1-ol $(25.3 \mathrm{mmol}, 1 \mathrm{eq})$ in $25 \mathrm{ml}$ benzene was added dropwise $3.3 \mathrm{~g}$ isocyanato-benzene $(27.9 \mathrm{mmol}, 1.1 \mathrm{eq})$. After stirring for $16 \mathrm{~h}$ at $\mathrm{rt}$ some preciptated $1,3-$ diphenyl-urea was removed by filtration. The filtrate was concentrated in vacuo and the residue was purified by flash chromatography on silica gel (5:1 cyclohexane:EtOAc). Compound 4 was obtained as a colorless oily liquid (5.40 g, 97.0\%).

${ }^{1} \mathrm{H}\left(300 \mathrm{MHz}, \mathrm{C}_{6} \mathrm{D}_{6}, \mathrm{ppm}\right) 7.38(\mathrm{~d}, J=7.9 \mathrm{~Hz}, 2 \mathrm{H}), 7.09$ (t, $\left.J=7.9 \mathrm{~Hz}, 2 \mathrm{H}\right), 6.84$ (t, $J=7.9 \mathrm{~Hz}$, 2H), 6.61 (br s, 1H), 6.60-5.78 (m, 1H), 5.10-4.90 (m, 2H), 4.03 (t, $J=6.6,2 \mathrm{H}), 1.88$ (q, $J=7.1$ $\mathrm{Hz}, 2 \mathrm{H}), 1.50-1.35(\mathrm{~m}, 2 \mathrm{H}), 1.33-1.18(\mathrm{~m}, 2 \mathrm{H})$

${ }^{13} \mathrm{C}\left(75.5 \mathrm{MHz}, \mathrm{C}_{6} \mathrm{D}_{6}, \mathrm{ppm}\right) \quad 153.8,138.9,138.5,128.3,123.3,118.8,114.9,65.0,33.5,28.6$, 25.4

HRMS (EI) calcd for $\mathrm{C}_{13} \mathrm{H}_{17} \mathrm{NO}_{2} 219.1260$ found $219.1259\left(\mathrm{M}^{+}\right)$

\section{2-Hydroxy-benzoic acid hex-5-enyl ester $(5)^{[4]}$}<smiles>C=CCCCCOC(=O)c1ccccc1O</smiles>

$\mathrm{C}_{13} \mathrm{H}_{16} \mathrm{O}_{3}$

Mol. Wt.: 220.26

To a suspension of $6.99 \mathrm{~g}$ 2-hydroxy-benzoic acid (50.6 mmol, $2.0 \mathrm{eq}$ ) in $50 \mathrm{ml} \mathrm{CH}_{2} \mathrm{Cl}_{2}$ was added $3.09 \mathrm{~g}$ dimethyl-pyridin-4-yl-amine (25.3 mmol, $1.0 \mathrm{eq})$, followed by $2.53 \mathrm{~g}$ hex-5-en-1-ol (25.3 mmol, $1 \mathrm{eq})$ and $7.85 \mathrm{~g}$ dicyclohexyl-carbodiimide (63.2 mmol, $2.5 \mathrm{eq})$. The suspension was stirred for $3 \mathrm{~d}$ and then filtrated. The filtrate was concentrated in vacuo and the residue was purified by chromatography on silica gel (2:1 hexanes: $\left.\mathrm{CH}_{2} \mathrm{Cl}_{2}\right)$. Compound 4 was obtained as a colorless oily liquid $(5.51 \mathrm{~g}, 98.8 \%)$.

${ }^{1} \mathrm{H}\left(300 \mathrm{MHz}, \mathrm{C}_{6} \mathrm{D}_{6}, \mathrm{ppm}\right) \quad 10.72(\mathrm{~s}, 1 \mathrm{H}), 7.74(\mathrm{dd}, J=8.0,1.7 \mathrm{~Hz}, 1 \mathrm{H}), 7.35$ (td, $J=7.8,1.7$ $\mathrm{Hz}, 1 \mathrm{H}), 6.88(\mathrm{dd}, J=8.4 \mathrm{~Hz}, 1 \mathrm{H}), 6.81$ (td, $J=7.6,1.0 \mathrm{~Hz}, 1 \mathrm{H}), 5.80-5.65(\mathrm{~m}, 1 \mathrm{H}), 5.00-4.85$ $(\mathrm{m}, 2 \mathrm{H}), 4.26(\mathrm{t}, J=6.6 \mathrm{~Hz}, 2 \mathrm{H}), 2.04(\mathrm{q}, J=7.1 \mathrm{~Hz}, 2 \mathrm{H}), 1.78-1.64(\mathrm{~m}, 2 \mathrm{H}), 1.53-1.39(\mathrm{~m}, 2 \mathrm{H})$ 
${ }^{13} \mathrm{C}\left(100.6 \mathrm{MHz}, \mathrm{CDCl}_{3}, \mathrm{ppm}\right) \quad 170.2,161.8,138.2,135.6,129.9,119.1,117.6,115.0,112.7$, $65.3,33.3,28.0,25.3$

HRMS (EI) calcd for $\mathrm{C}_{13} \mathrm{H}_{16} \mathrm{NO}_{3} 220.1099$ found $220.1099\left(\mathrm{M}^{+}\right)$

CHN calcd C: 70.89, H: 7.32 found C: 70.68 , H: 7.40

\section{General procedure for the hydroformylation of 1-octene}

To a solution of $1.8 \mathrm{mg}(6.98 \mu \mathrm{mol})\left[\mathrm{Rh}(\mathrm{CO})_{2} \mathrm{acac}\right]$ in $24 \mathrm{ml}$ toluene in a schlenk tube was added $140 \mu \mathrm{mol}$ of the particular ligand (see below and table 1). Subsequently an aliquot of $3 \mathrm{ml}(0.87$ $\mu \mathrm{mol}[\mathrm{Rh}]$-catalyst, $1 \mathrm{eq}$ ) of the above prepared catalyst solution was injected in each of the 8 reaction vessels. Catalyst preformation was done at $5 \mathrm{bar}\left(\mathrm{CO} / \mathrm{H}_{2}, 1: 1\right)$ and $90{ }^{\circ} \mathrm{C}$ for $30 \mathrm{~min}$. The reaction vessels were adjusted to the appropriate reaction temperatures and pressurized to 8 bar $\left(\mathrm{CO} / \mathrm{H}_{2}, 1: 1\right)$. Under these conditions each vessel was loaded with $0.68 \mathrm{~g}(6.1 \mathrm{mmol}, 7000 \mathrm{eq}) 1$ octene and additional $0.5 \mathrm{ml}$ toluene. The pressure was adjusted to $10 \mathrm{bar}\left(\mathrm{CO} / \mathrm{H}_{2}, 1: 1\right)$ for each vessel. Pressure and temperature were kept constant over the complete reaction time. After $4 \mathrm{~h}$ the reaction vessels were cooled down to $\mathrm{rt}$, depressurized and the solutions were analyzed via GC.<smiles>CC(C)(C)c1cc(-c2ccccc2)c2c(c1)C(C)(C)c1cc(C(C)(C)C)cc(-c3ccccc3)c1O2</smiles>

$\mathrm{C}_{23} \mathrm{H}_{32} \mathrm{OP}_{2}$

Mol. Wt.: 386,45

\section{General procedure for the hydroformylation of functionalized terminal alkenes}

Hydroformylations of functionalized olefines were performed each with in situ prepared [Rh]catalyst modified with the ligands TPP and 6-DPPon (3), respectively.

To a solution of $1.8 \mathrm{mg}(6.98 \mu \mathrm{mol}, 1 \mathrm{eq})\left[\mathrm{Rh}(\mathrm{CO})_{2} \mathrm{acac}\right]$ in $10 \mathrm{ml}$ toluene in a schlenk tube was added $140 \mu \mathrm{mol}(20 \mathrm{eq}$ ) of the particular ligand. The solution was stirred for $5 \mathrm{~min}$ and then charged with $6.98 \mathrm{mmol}$ (1000 eq) of alkenic substrate. The solution was transferred to the autoclave via syringe, pressurized to $10 \mathrm{bar}\left(\mathrm{CO} / \mathrm{H}_{2}, 1: 1\right)$ and heated to $70{ }^{\circ} \mathrm{C}$ in an oil bath. After $20 \mathrm{~h}$ the autoclave was cooled down to $\mathrm{rt}$ and depressurized. The $l / b$-ration was analyzed via ${ }^{1} \mathrm{H}-$ NMR spectra or GC analysis of the crude reaction mixture. The solution was filtrated over a plug of silica $(5 \times 2 \mathrm{~cm})$ with $50 \mathrm{ml}$ AcOEt and concentrated in vacuo. The conversion was always 
quantitative, the isolated yields were typically in the range of 90 to $95 \%$ and the products obtained were essentially pure. For the aldehydes 7-bromo-heptanal ${ }^{[5]}$ (table 2: entry 1), acetic acid 7-oxo-heptyl ester ${ }^{[6]}$ (table 2: entry 2), 12-oxo-dodecanoic acid methyl ester ${ }^{[7]}$ (table 2: entry 3), 6-oxo-heptanal ${ }^{[8]}$ (table 2: entry 4), 4-hydroxy-butyraldehyde (tetrahydro-furan-2-ol) ${ }^{[9]}$ (table 2: entry 7) and 12-hydroxy-dodecanal ${ }^{[10]}$ (table 2: entry 8) the spectral and analytical data correspond to those reported previously. The hydroformylation products derived from phenylcarbamic acid hex-5-enyl ester (4) and 2-hydroxy-benzoic acid hex-5-enyl ester (5) have not been reported in literature before. The corresponding aldehydes were purified by flash chromatography on silica gel (cyclohexane/AcOEt 1:1) to afford analytically pure samples.

\section{Phenyl-carbamic acid 6-oxo-hexyl ester (6)}

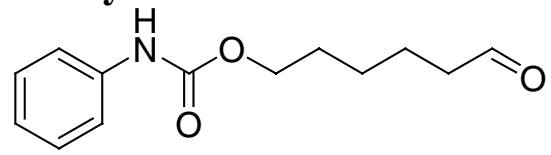

$\mathrm{C}_{13} \mathrm{H}_{17} \mathrm{NO}_{3}$

Mol. Wt.: 235.28

Compound 6 was obtained as a white solid (1.50 g, 91.4\%).

$\operatorname{mp} 51^{\circ} \mathrm{C}$

${ }^{1} \mathrm{H}\left(400 \mathrm{MHz}, \mathrm{CDCl}_{3}, \mathrm{ppm}\right) \quad 9.76(\mathrm{t}, J=1.6 \mathrm{~Hz}, 1 \mathrm{H}), 7.40-7.34(\mathrm{~m}, 2 \mathrm{H}), 7.32-7.26(\mathrm{~m}, 2 \mathrm{H})$, 7.08-7.02 (m, 1H), 6.61 (br s, 1H), 4.15 (t, $J=6.7 \mathrm{~Hz}, 2 \mathrm{H}), 2.43(\mathrm{td}, J=7.3,1.7 \mathrm{~Hz}, 2 \mathrm{H}), 1.73-$ $1.60(\mathrm{~m}, 4 \mathrm{H}), 1.47-1.32(\mathrm{~m}, 4 \mathrm{H})$

${ }^{13} \mathrm{C}\left(100.6 \mathrm{MHz}, \mathrm{CDCl}_{3}, \mathrm{ppm}\right) \quad 202.6,152.9,138.1,129.1,125.5,118.7,65.2,43.9,28.8,25.8$, 22.0

CHN calcd C: $67.45, \mathrm{H}: 7.68, \mathrm{~N}: 5.62$ found C: $67.42, \mathrm{H}: 7.77, \mathrm{~N}: 5.55$

\section{2-Hydroxy-benzoic acid 7-oxo-heptyl ester (7)}<smiles>O=CCCCCCCOC(=O)c1ccccc1O</smiles>

$\mathrm{C}_{14} \mathrm{H}_{18} \mathrm{O}_{4}$

Mol. Wt.: 250.29

Compound 7 was obtained as a white solid (1.53 g, 87.4\%).

$\operatorname{mp} 34{ }^{\circ} \mathrm{C}$

${ }^{1} \mathrm{H}\left(300 \mathrm{MHz}, \mathrm{CDCl}_{3}, \mathrm{ppm}\right) \quad 10.80(\mathrm{~s}, 1 \mathrm{H}), 9.76(\mathrm{t}, J=1.8 \mathrm{~Hz}, 1 \mathrm{H}), 7.82(\mathrm{dd}, J=7.9,1.7 \mathrm{~Hz}$, 1H), $7.44(\mathrm{td}, J=7.9,1.7 \mathrm{~Hz}, 1 \mathrm{H}), 6.96(\mathrm{~d}, J=7.8 \mathrm{~Hz}, 1 \mathrm{H}), 6.87(\mathrm{td}, J=7.6,1.0 \mathrm{~Hz}, 1 \mathrm{H}), 4.34(\mathrm{t}$, 
$J=6.6 \mathrm{~Hz}, 2 \mathrm{H}), 2.44(\mathrm{td}, J=7.0,1.8 \mathrm{~Hz}, 2 \mathrm{H}), 1.86-1.73(\mathrm{~m}, 2 \mathrm{H}), 1.72-1.60(\mathrm{~m}, 2 \mathrm{H}), 1.55-1.34$ $(\mathrm{m}, 2 \mathrm{H})$

${ }^{13} \mathrm{C}\left(75.5 \mathrm{MHz}, \mathrm{CDCl}_{3}, \mathrm{ppm}\right) \quad 202.4,170.2,161.7,135.6,129.8,119.1,117.6,112.6,65.2,43.7$, $28.7,28.4,25.8,21.9$

HRMS (EI) calcd for $\mathrm{C}_{14} \mathrm{H}_{18} \mathrm{NO}_{4} 250.1208$ found $220.1205\left(\mathrm{M}^{+}\right)$

CHN calcd C: 67.18, H: 7.25 found C: 67.17, H: 7.25

\section{[cis-PtCl 2 (6-DPPon $\left.)_{2}\right](8)$}

To a solution of $68.4 \mathrm{mg}(182 \mu \mathrm{mol}, 1 \mathrm{eq})\left[\right.$ cis- $\left.\mathrm{PtCl}_{2}(\mathrm{COD})_{2}\right]$ in $2.5 \mathrm{ml} \mathrm{CH}_{2} \mathrm{Cl}_{2}$ was added 102 mg (366 $\mu \mathrm{mol}, 2$ eq) 6-diphenylphosphanyl- $1 H$-pyridin-2-one (3). The citreous suspension was diluted with additional $2.5 \mathrm{ml} \mathrm{CH} \mathrm{Cl}_{2}$ and the yellow solution was stirred for $5 \mathrm{~min}$. The solvent was removed in vacuo and the residue was washed two times with pentane. The remaining white solid was dried in oil pump vacuo. Suitable crystals for $\mathrm{x}$-ray analysis were grown from a solution of $20 \mathrm{mg}$ [cis- $\mathrm{PtCl}_{2}$ (6-DPPon) $)_{2}$ in $1 \mathrm{ml} \mathrm{CH}_{2} \mathrm{Cl}_{2}$ at $\mathrm{rt}$.

\section{Literature}

[1] Brown, H. C.; Lynch, G. C. J. Org. Chem. 1981, 46(3), 531-538.

[2] Strother, T.; Cai, W.; Zhao, X.; Hamers, R. J.; Smith, L. M. J. Amer. Chem. Soc. 2000, 122(6), 12051209.

[3] Still, W. C.; Kahn, M.; Mitra, A. J. Org. Chem. 1978, 43, 2923-2925.

[4] Höfle, G.; Steglich, W.; Vorbrüggen, H. Angew. Chem. 1978, 90, 602-615.

[5] Hester, J. B.; Gibson, J. K.; Buchanan, L. V.; Cimini, M. G.; Clark, M. A.; Emmert, D. E.; Glavanovich, M. A.; Imbordino, R. J.; LeMay, R. J.; McMillan, M. W.; Perricone, S. C.; et al. J. Med. Chem. 2001, 44(7), 1099-1115.

[6] Muchowski, J. M.; Venuti, M. C. J. Org. Chem. 1981, 46(2), 459-461.

[7] Myers, A. G.; Gin, D. Y.; Rogers, D. H. J. Amer. Chem. Soc. 1994, 116(11), 4697-4718.

Baldwin, J. E.; Adlington, R. M.; Ramcharitar, S. H. Tetrahedron 1992, 48(14), 2957-2976.

[8] Shin, H. S.; Lee, C. W.; Lee, J. Y.; Huh, T. S. Eur. J. Org. Chem. 2000, 2, 335 - 348.

[9] Bergman, N.-A.; Halvarsson, T. J. Org. Chem. 1988, 53(11), 2548-2552.

Chickos, J. S.; Uang, J. Y.-J.; Keiderling, T. A. J. Org. Chem. 1991, 56(7), 2594-2596.

Nakano, T.; Terada, T.; Ishii, Y.; Ogawa, M. Synthesis 1986, 9, 774-776.

[10] Cuny, G. D.; Buchwald, S. L. J. Amer. Chem. Soc. 1993, 115(5), 2066-2068. 